\title{
Pour une géographie du cosmopolitisme autour du boulevard Saint-Laurent (Montréal)
}

Mises en récit, nostalgies patrimoniales et constructions territoriales

\section{Marie-Laure Poulot}

\section{OpenEdition}

\section{Journals}

Édition électronique

URL : http://journals.openedition.org/cdg/336

DOI : $10.4000 /$ cdg.336

ISSN : 2107-7266

\section{Éditeur}

UMR 245 - CESSMA

Référence électronique

Marie-Laure Poulot, «Pour une géographie du cosmopolitisme autour du boulevard Saint-Laurent (Montréal) », Carnets de géographes [En ligne], 8 | 2015, mis en ligne le 01 septembre 2015, consulté le 24 septembre 2020. URL : http://journals.openedition.org/cdg/336 ; DOI : https://doi.org/10.4000/ cdg.336

Ce document a été généré automatiquement le 24 septembre 2020

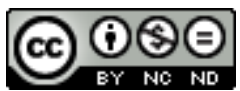

La revue Carnets de géographes est mise à disposition selon les termes de la Licence Creative Commons Attribution - Pas d'Utilisation Commerciale - Pas de Modification 4.0 International. 


\title{
Pour une géographie du cosmopolitisme autour du boulevard Saint-Laurent (Montréal)
}

Mises en récit, nostalgies patrimoniales et constructions territoriales

\author{
Marie-Laure Poulot
}

Cette thèse de géographie porte sur la construction d'une géographie $d u$ cosmopolitisme appliquée au boulevard Saint-Laurent à Montréal. Main Street longue de six kilomètres, le boulevard s'impose à la fois comme une coupure entre les deux «solitudes", française et anglaise, mais aussi comme le corridor d'accueil des différentes communautés immigrées installées à Montréal durant le XXe siècle. Elle relie ainsi différents lieux du cosmopolitisme, avec tous les effets de contrastes, de complémentarités et d'hybridations possibles. La thèse interroge la production de l'espace urbain au prisme de ce cosmopolitisme, entendu comme projet politique, stratégie économique, mais aussi comme sociabilité habitante. 


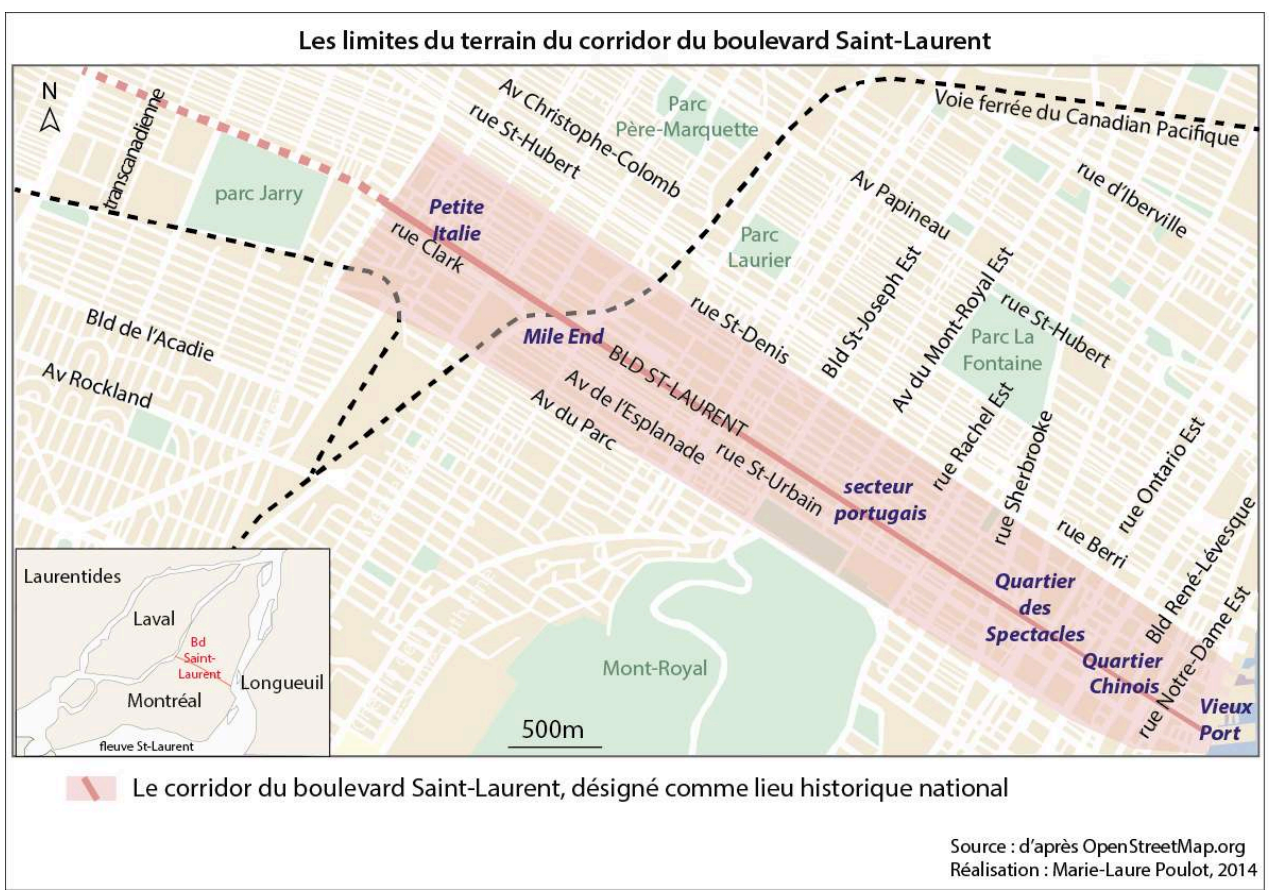

2 Cette thèse fait le choix du concept de cosmopolitisme en lieu et place de celui de multiculturalisme: quand ce dernier offre une conception de la société comme un ensemble donné de communautés culturelles préconstituées et cloisonnées, la vision " cosmopolite " valorise à rebours l'affiliation volontaire et révocable des individus à une pluralité de groupes aux frontières mouvantes.

Les études sur la diversité s'attachent plutôt à la ville dans son ensemble ou au quartier et à ses espaces publics où peuvent se croiser, voire interagir les individus; il en est de même du quartier de la gare, ou des marchés. Mon choix s'est porté sur une rue en ce qu'elle constitue un espace à la jonction entre ces différents lieux du cosmopolitisme : plus qu'une simple juxtaposition de quartiers ethniques ou multiethniques, l'étude de la rue en tant qu'axe de mise en continuité spatiale conduit à envisager les ruptures, les mises en relation, les différentes échelles spatiales et temporelles; elle est aussi le lieu d'un cosmopolitisme vécu, mêlant récits individuels et collectifs, marchandisation et appropriation.

Deux grandes questions ont guidé le travail : comment les différents acteurs dans la ville utilisent les images et récits urbains cosmopolites pour aménager, transformer, recréer, voire faire de la ville? Dans quelle mesure la construction d'une mémoire cosmopolite de la ville tient compte des représentations et pratiques des habitants et des impératifs internationaux de positionnement dans la hiérarchie urbaine internationale? La thèse est organisée autour des trois grandes dimensions du cosmopolitisme : un cosmopolitisme comme projet identitaire et politique inscrit dans la continuité de l'histoire montréalaise, un cosmopolitisme instrumentalisé comme marchandise dans des stratégies de branding (notamment au sein des quartiers ethniques ou du quartier des Spectacles), et enfin, un cosmopolitisme vécu, pratiqué et raconté par les habitants.

Pour mener ce travail, j'ai adopté une méthode «sensible» et qualitative fondée notamment sur la marche, à la fois solitaire, «accompagnée » et collective. Ces trois formes de marches ont révélé combien le contact avec les lieux conduit à une immédiateté, un lien direct avec le temps et l'espace. La marche m'a conduite à 
réfléchir sur cette pratique au sein même des ré-investissements individuels et collectifs des citadins par rapport au boulevard. Dans une volonté d'interroger les mises en récit des résidents et usagers, plus d'une centaine d'entretiens ont été réalisés avec des habitants, acteurs institutionnels, associatifs, commerçants, etc. De surcroît, j'ai mené des observations centrées sur le paysage urbain, lors de «lieux-moments » particuliers (fêtes, visites urbaines), mais aussi lors des consultations publiques. Articles de presse, rapports et autres documents provenant d'institutionnels, de promoteurs ou encore d'associations ont également été collectés et utilisés. Enfin, une trentaine de romans et autres textes littéraires considérés comme mode d'appréhension alternatif de la réalité géographique ont complété le matériau d'étude. Dans cette approche, j'ai tenté de tenir ensemble plusieurs échelles, tant du linéaire du boulevard que de son épaisseur, celle de la rue dans son entier, celles de microquartiers ethniques ou multiethniques (Quartier chinois, Petite Italie, Mile End), ou encore celles de lieux ponctuels (commerces, restaurants ou encore institutions).

Cette recherche montre que le cosmopolitisme du boulevard Saint-Laurent appelle à dépasser l'opposition binaire entre des valeurs universelles et repli sur soi, ou entre cosmopolitisme des élites cultivées et mobiles et l'esprit de clocher d'habitants locaux. Il s'apparente à un cosmopolitisme enraciné dans le local, avec des réappropriations d'apports venus d'ailleurs. En outre, malgré le déclin des différentes communautés, les récits actuels réinvestissent les anciennes narrations, véritables "géographies imaginaires et imaginées » de la ville pour aboutir à une réinvention tant du boulevard que du cosmopolitisme qui lui est attaché.

7 La situation montréalaise révèle également une instrumentalisation politique et commerciale du cosmopolitisme à l'œuvre dans les constructions territoriales, avec un glissement de plus en plus net vers le culturel. Le thème de la "créativité ", conçu comme plus consensuel, tend à recouvrir et à « invisibiliser » la présence des minorités ethnoculturelles. Enfin, si le cosmopolitisme, objet et sujet des représentations littéraires, touristiques et politiques, connait une traduction spatiale visible dans le paysage, il est aussi un élément intériorisé, vécu au quotidien au point que les citadins occupent une place centrale dans la construction de la "montréalité " cosmopolite. Cette thèse a d'ailleurs permis d'isoler un certain nombre de "figures cosmopolites »habitants, commerçants, acteurs commerciaux - qui racontent et vivent la diversité.

Enfin, le cosmopolitisme constitue bel et bien un objet dont peut se saisir la géographie. Devenu une réalité des grandes villes caractérisées par la diversité, le cosmopolitisme est éminemment spatial, au cœur des discours politiques et des réflexions sur l'aménagement du territoire avec tous les effets d'instrumentalisation inhérents. Les habitants doivent composer avec cette diversité ce qui les conduit à des emprunts, des adaptations, des évitements qui peuvent également se lire dans l'espace. Les mises en récits et en espaces du cosmopolitisme recouvrent ainsi pour une bonne part la production de l'espace comme espace pluriel dans lequel se rencontrent, se confrontent et parfois se superposent espace perçu, vécu et conçu (Lefebvre, 1974). La dimension marchande du cosmopolitisme met bien en lumière ces agencements complexes. L'opposition aux grandes chaînes commerciales et la nostalgie liée aux transformations du boulevard amènent certains habitants à rejeter le cosmopolitisme standardisé et marchandisé au profit d'une authenticité plus ou moins rêvée. Cette idée d'authenticité, liée à l'image du boulevard comme quartier ou comme " communauté ", est pourtant à son tour reprise par les acteurs du développement économique (les 
Sociétés de développement commercial notamment) et louée par les gentrifieurs, notamment dans le quartier du Mile End. Le cosmopolitisme utilisé à des fins économiques et touristiques dans les quartiers « conçus » interroge donc la production de la ville par les autorités et les acteurs économiques : l'analyse à grande échelle de plusieurs quartiers et tronçons de rue permet de souligner les résistances, tactiques de contournement et appropriations habitantes.

\author{
Discipline \\ Géographie

\section{Directeurs} \\ Marie-Hélène Bacqué et Philippe Gervais-Lambony

\section{Université} \\ Université Paris Ouest Nanterre la Défense
}

Membre du jury de thèse, soutenue le 1er décembre 2014

Marie-Hélène Bacqué, professeure, Université Paris Ouest Nanterre la Défense (directrice)

Guy Di Méo, professeur émérite, Université de Bordeaux Montaigne (examinateur) Annick Germain, Professeure, Institut national de la Recherche (INRS -

Urbanisation Culture Société) (rapportrice)

Philippe Gervais-Lambony, professeur, Université Paris Ouest Nanterre la Défense (directeur)

Sonia Lehman-Frisch, professeure, Université Paris Ouest Nanterre la Défense (présidente du jury)

Vincent Veschambre, professeur, École Nationale Supérieure d'Architecture de Lyon (rapporteur)

\title{
Situation professionnelle à l'issue de la thèse
}

ATER en géographie, Université de Cergy-Pontoise

\section{Courriel de l'auteur}

marielaure.poulot[at]yahoo.fr

\section{INDEX}

Thèmes : Carnets de soutenances 